\title{
Autologous PSMA-specific TGFb-resistant CAR T Cells
}

National Cancer Institute

\section{Source}

National Cancer Institute. Autologous PSMA-specific T GFb-resistant CAR T Cells. NCI

Thesaurus. Code C148496.

Autologous T-lymphocytes transduced with a lentiviral vector expressing a chimeric antigen receptor (CAR) consisting of an anti-prostate specific membrane antigen (PSMA) single chain variable fragment (scFv) and expressing a dominant negative (DN) form of transforming growth factor-beta (T GF-beta; T GFb) receptor, with potential immunomodulating and antineoplastic activities. Upon transfusion, the autologous PSMA-specific TGFb-resistant CAR T cells are directed to and induce selective toxicity in PSMA-expressing tumor cells. The tumor-associated antigen (TAA) PSMA is overexpressed by prostate cancers; its expression is associated with poor prognosis and metastasis. The inclusion of the DN TGFb receptor blocks signaling of the immunosuppressive cytokine T GFb in the tumor microenvironment (TME) and makes the CAR T cells resistant to T GFb. T GFb negatively regulates T-cell proliferation and activation and plays a key role in tumor immune suppression. 